\title{
Is There Benefit to Free Over Pedicled Vascularized Grafts in Augmenting Tibial Intercalary Allograft Constructs?
}

\author{
Marco Manfrini MD, Srimanth Bindiganavile MD, Ferhat Say MD, \\ Marco Colangeli MD, Laura Campanacci MD, Massimiliano Depaolis MD, \\ Massimo Ceruso MD, Davide Donati MD
}

Received: 7 June 2016/ Accepted: 29 November 2016/Published online: 19 December 2016

(C) The Association of Bone and Joint Surgeons $\mathbb{R} 2016$

\begin{abstract}
Background Intercalary reconstruction of tibial sarcomas with vascularized fibula autografts and massive bone allografts is reliable with predictable long-term results. However, inadequate data exist comparing free and pedicled vascularized fibula autografts in combination with a massive bone allograft in patients undergoing intercalary tibia reconstructions.

Questions/Purposes Among patients undergoing largesegment intercalary allografting, we sought to compare supplemental free vascularized fibular autografts with supplemental pedicled vascularized fibular autografts, in terms of (1) oncologic results, (2) complications associated
\end{abstract}

This study was supported by Italian Ministry of Health (Grant Minsal RF-2010-2321501).

Each author certifies that he or she, or a member of his or her immediate family, has no funding or commercial associations (eg, consultancies, stock ownership, equity interest, patent/licensing arrangements, etc) that might pose a conflict of interest in connection with the submitted article.

All ICMJE Conflict of Interest Forms for authors and Clinical Orthopaedics and Related Research ${ }^{\circledR}$ editors and board members are on file with the publication and can be viewed on request.

Each author certifies that his or her institution approved or waived approval for the human protocol for this investigation and that all investigations were conducted in conformity with ethical principles of research.

This study was performed at Oncology Department III, Rizzoli Orthopedic Institute, Bologna, Italy.

M. Manfrini, M. Colangeli, L. Campanacci, M. Depaolis,

D. Donati

Department of Orthopedic Oncology, Unit III, Istituto

Ortopedico Rizzoli, Via Pupilli 1, 40136 Bologna, Italy

S. Bindiganavile $(\square)$

Department of Musculoskeletal Oncology, Manipal Hospital,

\#98, HAL Airport Road, Bengaluru 560017, India

e-mail: srimanth.dr@gmail.com with surgery, (3) Musculoskeletal Tumor Society (MSTS) scores, and (4) surgical time.

Methods Between 1994 and 2013, we treated 320 patients, younger than 40 years, with tibial sarcomas. Thirty-five patients $(11 \%)$ underwent amputations. One hundred ninetyfive patients (61\%) were treated with intraarticular resection of the tibia, which constituted 104 tumor endoprostheses, 63 proximal tibia allograft prosthetic composites, 21 osteoarticular allografts, and seven arthrodeses with allografts. Ninety patients $(28 \%)$ underwent joint-sparing intercalary reconstruction. Forty-one (13\%) of these 90 patients were treated with allografts alone, two (1\%) with vascularized fibula grafts, and $47(15 \%)$ with intercalary allografts supplemented by autografts (free fibular autografts, 22 patients, 7\%; pedicled fibular autografts, 25 patients, $8 \%$ ). During the study period, we used free vascularized fibular autografts in association with massive bone allograft for a resection longer than $12 \mathrm{~cm}$ with a very small periarticular residual segment. The choice for using a pedicled fibula harvested in the ipsilateral leg initially was for patients having only diaphyseal resections and the indication was later extended to intraepiphyseal osteotomies with a small periarticular residual segment. The goals of this study are to present the long-term results in this group of patients and compare their results based on the type of vascularized fibula harvest. There were 33 male and 14 female patients with mean age of $14 \pm 6$ years. The median followup was 84 months

\footnotetext{
F. Say

Department of Orthopaedics and Traumatology, Faculty of Medicine, Ondokuz Mayıs University, 55139 Samsun, Turkey

M. Ceruso

Department of Hand Surgery, Azienda Ospedaliare-Universitaria Careggi, 50139 Florence, Italy
} 
(range, 7-231 months). No patients were lost to followup before 1 year. Four patients died and were not available for followup after 18 months. The mean tibia resection length was $15 \pm 4 \mathrm{~cm}$ and mean length of the harvested vascularized fibula was $18 \pm 4 \mathrm{~cm}$.

Results Overall 5- and 10-year oncologic survival rates in this study were $87 \% \pm 5 \%$ and $83 \% \pm 6 \%$ respectively. With the numbers available, we observed no difference in survivorship free from death from disease between the study groups $(85 \% \pm 8 \%$ [95\% CI, 174-232 months] of the free vascularized group versus $82 \% \pm 8 \%$ [95\% CI, 148206 months] of the pedicled fibula graft group; $\mathrm{p}=0.741$ ). At last followup, 40 patients had no evidence of disease and seven had died of disease. Local recurrence was observed in two patients in the supplemental free vascularized fibula group and three patients in the supplemental pedicled vascularized fibula group, whereas metastases was observed in eight patients. With the numbers available, we observed no difference in the proportion of patients experiencing surgical complications between those treated with free vascularized fibula grafts and those treated with pedicled grafts (eight of 22 [36\%] versus nine of 25 [36\%] respectively; $\mathrm{p}=0.605)$. With the numbers available, we observed no difference in mean MSTS scores between patients treated with free vascularized fibula grafts and those treated with pedicled grafts $(24 \pm 9$ versus $25 \pm 8$; mean difference, 0.48 ; 95\% CI, 0.54-4.6; $\mathrm{p}=0.858$ ). Mean surgical time was longer in the free vascularized fibula and massive bone allograft group at $9.4 \pm 1.7$ hours compared with that of the pedicled vascularized fibula and massive bone allograft group at $5.7 \pm 1.3$ hours (mean difference, 3.73 hours; 95\% CI, 2.8-4.6 hours; $\mathrm{p} \leq 0.001$ ).

Conclusions Intercalary reconstruction of tibia sarcomas with massive bone allografts supplemented with vascularized fibula grafts provide predictable results. Complications occur as expected in a biologic reconstruction, but are salvageable, preserving the original construct. The pedicled fibula can be an alternative to a free contralateral fibula for intraepiphyseal resections. Comparative technical ease, shorter surgical time, avoidance of additional microvascular anastomosis, and avoidance of surgery on the contralateral leg are notable advantages of pedicled vascularized fibula over free fibula grafts to supplement allografts when indicated in intercalary tibia resections.

Level of Evidence Level III, therapeutic study.

\section{Introduction}

Limb-salvage surgery currently is performed in $85 \%$ to $90 \%$ of patients with malignant bone tumors [33]. Advances in imaging techniques permit an accurate preoperative determination of the tumor extent in metadiaphyseal locations, thereby allowing intercalary resection and joint preservation in selected cases, which may result in superior function [27]. Intercalary reconstruction of the tibia is performed either by biologic or synthetic means. Biologic reconstruction includes massive bone allograft, autograft (vascularized and nonvascularized), reimplantation of either irradiated or pasteurized tumor graft, or bone transport with an external fixator. Synthetic options include intercalary prostheses and acrylic cement that can be used as temporary spacers or palliation [18]. Massive bone allograft and vascularized fibula graft, either alone or combined, are common biologic sources for reconstruction of intercalary defects after tumor resection, either as a primary or a salvage procedure [7]. When used alone, massive bone allografts are associated with complications such as nonunion at the host-allograft junction, allograft fracture, and infection [3, 5, 17, 32]; by contrast, vascularized fibula grafts used alone or supplemental are associated with longer surgical times, risk of donor site morbidity, lengthy times to hypertrophy, and a high frequency of stress fracture [19]. Some studies have suggested that the shortcomings of each technique can be mitigated by using intercalary allografts in conjunction with a vascularized fibula autograft in primary and salvage situations, where immediate mechanical support is provided by the massive bone allograft allowing biologic activity of the vascularized fibula graft to result in hypertrophy and incorporation [7, 8]. Ozaki et al. [29] modified this technique by using an ipsilateral pedicle vascularized fibular autograft in the allograft shell for reconstruction of the tibia.

However, there is a paucity of evidence pertaining to indications for free or pedicled vascularized fibula grafts in combination with massive bone allograft in tibia intercalary reconstruction $[2,4,6,7]$. Furthermore, there is only one study which compares free and pedicled vascularized fibula grafts alone [6], but to our knowledge, there are no comparative studies regarding outcomes between the vascularized fibula groups when used with massive bone allograft in tibia reconstructions.

Among patients undergoing intercalary reconstruction with combined massive bone allograft and vascularized fibula, we sought to analyze overall results and compare the supplemental vascularized fibula autografts (pedicled and free) in terms of (1) oncologic results, (2) complications associated with surgery, (3) Musculoskeletal Tumor Society (MSTS) scores, and (4) surgical time.

\section{Methods}

Study Design and Setting

Between 1994 and 2013, we treated 320 patients, younger than 40 years, with tibial sarcomas. Thirty-five patients (11\%) underwent amputation. One hundred ninety-five 
patients $(61 \%)$ were treated with intraarticular resection of the tibia, which constituted 104 tumor endoprostheses, 63 proximal tibia allograft prosthetic composites, 21 osteoarticular allografts, and seven arthrodeses with allografts. Ninety patients $(28 \%)$ underwent joint-sparing intercalary reconstruction. Forty-one (13\%) of these 90 patients were treated with allografts alone, two $(1 \%)$ with only vascularized fibula grafts, and 47 (15\%) with intercalary allografts supplemented by autografts (free fibular autografts, 22, 7\%; pedicled fibular autografts, 25, 8\%). Patient data were retrieved from the electronic medical records system at the study institution, and no ethical committee approval was needed per institutional rules.

\section{Participants/Study Subjects}

The study group of 47 patients comprised 22 who underwent free vascularized fibula and massive bone allograft reconstruction and 25 who underwent pedicled vascularized fibula and massive bone allograft reconstruction. The mean age of the patients was $14 \pm 6$ years (range, 6-38 years). Thirtythree patients were male and 14 were female. Osteosarcoma was the most frequent diagnosis in 28 patients, followed by Ewing's sarcoma in 15, and adamantinoma in four. The proximal metadiaphysis was involved in 24 patients, tibia diaphysis in 19, and distal metadiaphysis in four. Forty patients (25 with osteosarcoma and 15 with Ewing's sarcoma) received neoadjuvant chemotherapy and one patient with Ewing's sarcoma of the tibial diaphysis received preoperative radiotherapy. Neoadjuvant chemotherapy according to protocols used at the time of treatment was provided to all patients except for three with low-grade osteosarcoma and four with adamantinoma [15, 16] (Table 1). The median followup was 84 months (range, 7231 months). No patients were lost to followup before 1 year, however four patients (two each in the free vascularized fibula group and pedicled vascularized fibula group) died and therefore were not available for followup after 18 months, hence the shortest followup of 7 months. All patients except the four who were deceased and one lost to followup after 53 months underwent clinical and radiologic assessments at least once during the last 5 years of the study period. The mean tibia resection length was $15 \pm 4 \mathrm{~cm}$ and mean length of the harvested vascularized fibula was $18 \pm 4 \mathrm{~cm}$. Blood products were transfused in 43 patients (data are not available for four patients), with a mean of $4 \pm 2$ units of blood transfused per patient.

\section{Description of Experiment, Treatment, or Surgery}

Since the early part of the study period, young patients with a diagnosis of metadiaphyseal sarcoma of the tibia with a

Table 1. Patient demographics

\begin{tabular}{|c|c|c|c|}
\hline Variable & $\begin{array}{l}\text { Free vascularized fibula-massive } \\
\text { bone allograft group } \\
(\mathrm{n}=22)\end{array}$ & $\begin{array}{l}\text { Pedicled vascularized fibula-massive } \\
\text { bone allograft group } \\
(\mathrm{n}=25)\end{array}$ & $\mathrm{p}$ Value \\
\hline Age (years) & $13.27 \pm 4.2$ & $14.96 \pm 7.2$ & 0.338 \\
\hline Gender & & & 0.775 \\
\hline Male & 15 & 18 & \\
\hline Female & 7 & 7 & \\
\hline Diagnosis & & & 0.459 \\
\hline Osteosarcoma & 15 & 13 & \\
\hline Ewing's sarcoma & 6 & 9 & \\
\hline Adamantinoma & 1 & 3 & \\
\hline Location & & & 0.003 \\
\hline Proximal tibia & 16 & 8 & \\
\hline Tibia diaphysis & 3 & 16 & \\
\hline Distal tibia & 3 & 1 & \\
\hline Neoadjuvant chemotherapy ${ }^{\S}$ & 20 & 20 & 0.423 \\
\hline Radiotherapy & $1 *$ & 0 & 0.468 \\
\hline Intraepiphyseal osteotomy & & & 0.001 \\
\hline Proximal & 15 & $6^{\dagger}$ & \\
\hline Distal & 3 & 1 & \\
\hline
\end{tabular}

$\mathrm{p}<0.005$ = significant; ${ }^{\S}$ not provided for seven patients (three had low-grade osteosarcoma and four had adamantinoma); *preoperative radiotherapy; ${ }^{\dagger}$ performed during latter part of study period. 

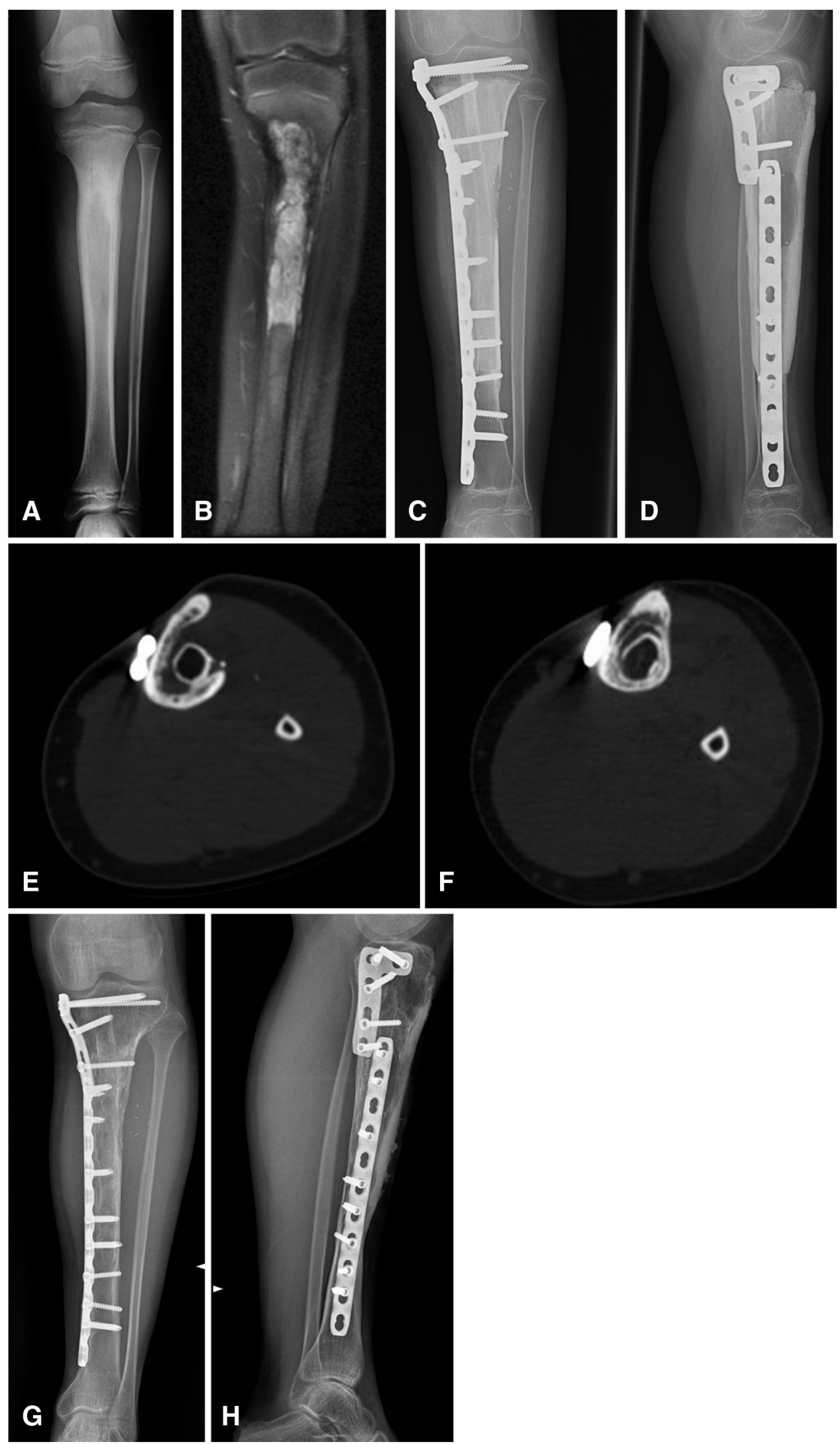
4Fig. 1A-H (A) A plain radiograph shows a Ewing's sarcoma of the proximal tibia diaphysis in an 11-year-old girl. (B) A representative coronal preoperative MR image of the tibia is shown. (C) AP and (D) lateral postoperative radiographs show the appearance 1 year after free vascularized fibula and massive bone allograft reconstruction. The oval slot for the fibula pedicle is evident on the lateral view radiograph. (E) An axial CT section shows the bony bridge between the inlaid fibula and allograft indicating progressive fusion at 4 years followup. (F) This axial CT section shows gradual incorporation of the free vascularized fibula with massive bone allograft at 9 years followup. (G) AP and (H) lateral radiographs obtained at the 10-year followup show the appearance of the free vascularized fibula with massive bone allograft. Incorporation of the vascularized fibula graft with allograft is observed by an indistinct appearance of the fibula graft.

good or favorable prognosis underwent intercalary or intraepiphyseal resections at our center and were candidates for a biologic reconstruction involving massive bone allografts. A previous study of massive bone allografts alone [17] for resections longer than $12 \mathrm{~cm}$ indicated unsatisfactory union rates, which led to greater need of revision using vascularized fibula grafts, whereas with intercalary prostheses, the experience involved small numbers and the results were not encouraging. Massive bone allograft was supplemented with vascularized fibula graft for the following indications: a free vascularized fibula graft (contralateral) was used when a myocutaneous flap was required and for intraepiphyseal reconstruction during the early part of this study. A pedicled vascularized fibula graft was used for diaphyseal reconstructions during the early part of the study; the indication was later extended to metaphyseal and intraepiphyseal resections as we gained more experience and understood the limitations of the length of the vascular pedicle. Contraindications to pedicled grafts were when the primary leg was irradiated, if the biopsy site was too lateral inhibiting the posterolateral fibula approach, and in rare situations of extremely long resections of the tibia with just the two epiphyses preserved (to save the ipsilateral fibula for mechanical support). Contraindications for a fibula harvest (either free or pedicled vascularized) included a fractured fibula, infection, or evidence of vascular compromise. A preoperative CT angiogram of both legs was performed routinely to further aid the decision-making regarding the type of vascularized fibula graft to be harvested.

All of the patients in this study were treated with intercalary resections (metadiaphyseal) or intraepiphyseal resection reconstructions and none had osteoarticular reconstructions. Surgical margins in all the patients were reported as wide and adequate. After resection of the tumor by the primary team (MM, LC, MD, and DD) regardless of the type, the vascularized fibula graft was harvested by another surgeon (MC and $\mathrm{MM}$ ) via a posterolateral incision on the leg $3 \mathrm{~cm}$ longer than the length of the resection with an intact periosteal cuff and sufficient pedicle length. The tibiofibular syndesmosis was fixed with a screw when the remnant lower fibula was less than $6 \mathrm{~cm}$ to prevent angular deformity. A frozen allograft that was size-matched for the patient was cut to match the length of the resection and then the medullary canal was reamed to allow the vascularized fibula graft to fit in. The allograft was treated by soaking in rifampicin and saline solution. Using a high-speed burr, an oval defect was created in the allograft cortex, at the appropriate level, to allow passage of the fibular vascular pedicle. This free vascularized fibula and massive bone allograft construct then was slotted in both ends of the tibia, ensuring central placement of the vascularized fibula graft, and osteosynthesis was performed with a combination of plate and screws followed by microanastomosis in a terminal-terminal fashion between the fibular vessels and the anterior tibialis vessels of the recipient leg (Fig. 1).

For patients who were treated with a pedicled vascularized fibula graft, a graft with a periosteal cuff was harvested through the posterolateral approach in contrast to the anterior or medial approach used for resection. The dissected fibular flap pedicled on the proximal peroneal vessels was transferred behind remnants of the interosseous membrane to fill the tibial defect. A bone allograft matched for resection length was prepared, with a complete, long posterior window, and was "wrapped" around the centrally placed vascularized fibula graft to support the grafted fibula against longitudinal stress. An osteosynthesis was done using a combination of plates and screws (Fig. 2).

During the study period, only three patients with a tumor contained in the diaphysis were not considered candidates for a pedicled vascularized fibula graft because one had a preoperative ipsilateral fracture of the fibula, one had received preoperative radiotherapy, and one had a large extraosseous mass in the posterolateral soft tissues. However, during the first 10 years of the study (1994-2003), 10 patients had their proximal tibia resected (nine by intraepiphyseal osteotomy) and only one patient had the proximal tibia reconstructed using an ipsilateral vascularized fibula graft while all the others received a free vascularized fibula harvested from the contralateral leg. During the second decade, of the 14 patients with proximal tibia resection, seven were treated with a free vascularized fibula graft and seven received a pedicled fibula graft to supplement the allograft. These differences were mainly attributable to the experience gained mobilizing the pedicled fibula graft for proximal tibia reconstruction.

\section{Aftercare}

Postoperatively, a cast was applied for 3 to 6 weeks, regardless of the osteosynthesis performed. This was followed by a short period of joint rehabilitation with the patient wearing a removable splint. Partial weightbearing 

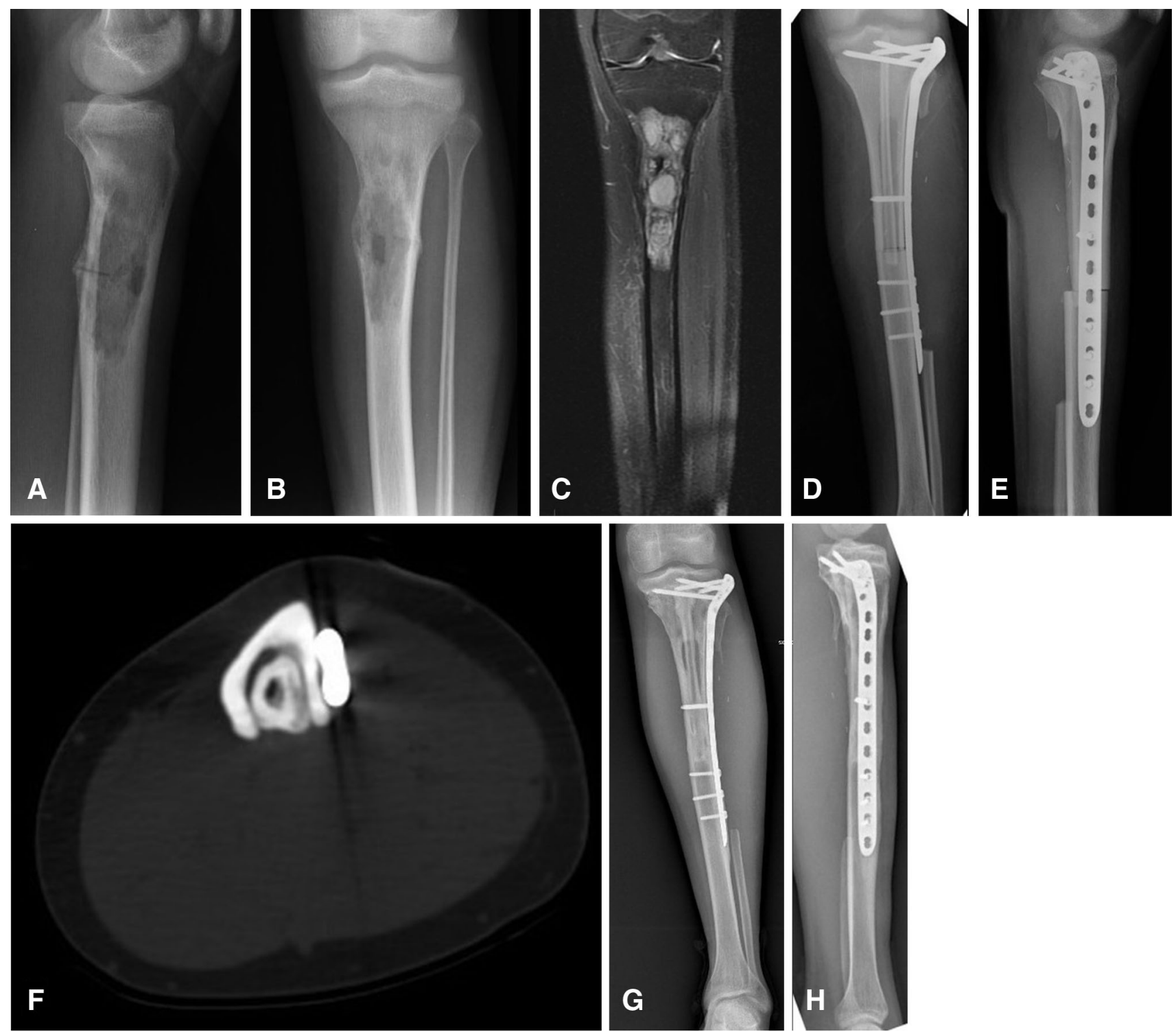

Fig. 2A-H (A) Lateral and (B) AP plain radiographs show the proximal tibia diaphysis involvement in a 17-year-old girl with osteosarcoma. (C) A coronal preoperative MR image of the tibia shows the extent of the tumor in the medullary cavity. Immediate postoperative (D) AP and (E) lateral view radiographs show the pedicled vascularized fibula and massive bone allograft construct. A long cortical window (lucent area in diaphysis) in the allograft to

was allowed as soon as union of the vascularized fibula graft on either junction was observed on radiographs, and was maintained until complete union of the vascularized fibula and massive bone allograft to host bone was evident, allowing full weightbearing.

\section{Followup Routine}

Surveillance for local and systemic recurrence consisted of clinical and radiologic assessments every 3 months for the accommodate the pedicled fibula graft is seen on the AP view. (F) An axial CT section shows gradual hypertrophy of the pedicled vascularized fibula inside the massive bone allograft at 3 years followup. (G) AP and (H) lateral view radiographs show the progressive incorporation of the pedicled fibula graft with allograft at 8 years postoperatively.

first 2 years, then every 6 months up to 5 years, and then annually thereafter. Although we supplemented allografts with free vascularized fibula grafts earlier during the period than pedicle fibula grafts, the followup periods were similar, with the free vascularized fibula group having an average of $111 \pm 59$ months compared with the pedicled vascularized fibula group at $88 \pm 65$ months (mean difference, 23 months; 95\% CI, 14.2-59.3 months; $\mathrm{p}=0.223$ ).

Followups consisted of clinical evaluation and radiology (radiographs of the limb and chest every 3 months, noncontrast CT scans of the chest and leg every 6 months, 
panoramic radiograph of both lower limbs in children). When a local recurrence was suspected clinically or radiologically, a contrast CT scan of the limb was performed followed by restaging with either a bone scan or 18-FDG positron emission tomography CT. The functional evaluation of the patients was done at the end of the followup using the modified 30-point MSTS score for the lower limb [14].

\section{Variables, Outcome Measures, Data Sources, and Bias}

Further evaluation for patients in the supplemental free vascularized fibula graft group (Table 2) and pedicled vascularized fibula graft group (Table 3) included retrieval of operative data such as length of tumor resection, type of vascularized fibula graft, length of harvested vascularized fibula graft, surgical time, blood transfusion, partial weightbearing walking, and time to full weightbearing. Assessment of radiologic union was determined using plain AP and lateral view radiographs of the surgically treated limb, which also were evaluated for graft incorporation, fracture of the allograft, and changes in the vascularized fibula (resorption/hypertrophy/fracture) at followups. Signs of progressive loss of the osteotomy line or haziness between the allograft and host tibia, cortical continuity, and haziness at the fibula-host bone junction were parameters to assess radiographic union. Owing to perceived limitations in assessment of the accurate time for radiographic union during followup, the mean time to full weightbearing ambulation on the affected limb was recorded to reflect the approximate time to union. Complications related to the procedure (neurovascular compromise, soft tissue failure, and infection) and graft-related complications (fracture of the allograft, fracture of the vascularized fibula graft, delayed union, nonunion, fracture, implant failure) with subsequent revision surgery were assessed. Regular CT of the surgically treated leg was performed every 6 months for the first 2 years, and once a year thereafter to evaluate the morphologic changes, incorporation, and failure of the vascularized fibula graft. Hypertrophy of the fibula was assessed on radiographs and CT scans with the following patterns: (1) When an allograft was intact, an increase in the diameter of the fibula without cortical thickening was observed; (2) when an allograft had a stress fracture, a substantial increase in the diameter of the medullary and cortical components of the fibula was observed in response to increased load on the fibula; (3) loss of viability of the vascularized fibula graft was the occurrence of progressive thinning or microfractures without subsequent healing $[9,25]$. Failure of the reconstruction was considered a change of the vascularized fibula and massive bone allograft construct with subsequent graft-implant revision.
Statistical Analysis, Study Size

Statistical analyses were performed using SPSS software, Version 21.0 (SPSS Inc, Armonk, NY, USA). Pearson chisquare evaluated by exact test was performed to observe any difference between the two groups. An independent ttest was performed to compare means between two groups. The Kaplan-Meier test was performed for survival analysis. A p value less than 0.05 was considered significant.

\section{Results}

Overall 5- and 10-year survival in this study was $87 \% \pm$ $5 \%$ and $83 \% \pm 6 \%$ respectively. With the numbers available, we observed no difference in survivorship free from death from disease between the study groups $(85 \% \pm 8 \%$ [95\% CI, 174-232 months] of the free vascularized group versus $82 \% \pm 8 \%$ [95\% CI, 148-206 months] of the pedicled fibula graft group; $p=0.741$ ). At last followup, 40 patients had no evidence of disease and seven had died of disease. Local recurrence was observed in five patients (two in the free fibula group and three in the pedicled fibula group). Of the five patients with relapse, the original response to chemotherapy was good ( $>90 \%$ of necrosis) in two, fair $(>80 \%,<90 \%)$ in one, and poor $(<80 \%)$ in two. With the numbers we had, we could not show a difference in local recurrence between the two groups of supplemental fibular grafts $(p=0.7)$. One patient with Ewing's sarcoma had a local recurrence, concurrent distant metastases, and died after second-line chemotherapy. Four patients with high-grade osteosarcoma had local recurrence (three at the level of the distal osteotomy and one in the soft tissue along the popliteal vessels). Three of these patients underwent amputation and one patient with recurrence at the level of the distal osteotomy site underwent removal of the whole biologic construct with adequate margins and had implantation of a modular proximal tibia tumor endoprosthesis. Metastases were observed in eight patients. Of these eight patients, one had metastases at diagnosis.

Overall in this study group of 47 patients, complications occurred in 17. They included delayed union at the osteotomy site (1), valgus deformity at the proximal tibia (2), varus deformity at the proximal tibia (1), macrofractures of the massive bone allograft- vascularized fibula construct (8, of which two were secondary to trauma) (Fig. 3), weakness of the extensor hallucis longus (1), deep infections (3), and implant allergy attributable to nickel (1). Sixteen additional procedures were performed to treat these complications: iliac crest autograft for delayed union (1), reosteosynthesis (10), removal of the implant owing to nickel allergy (1), change of the construct for deep infection (2), débridement for deep infection (1), and tendon 


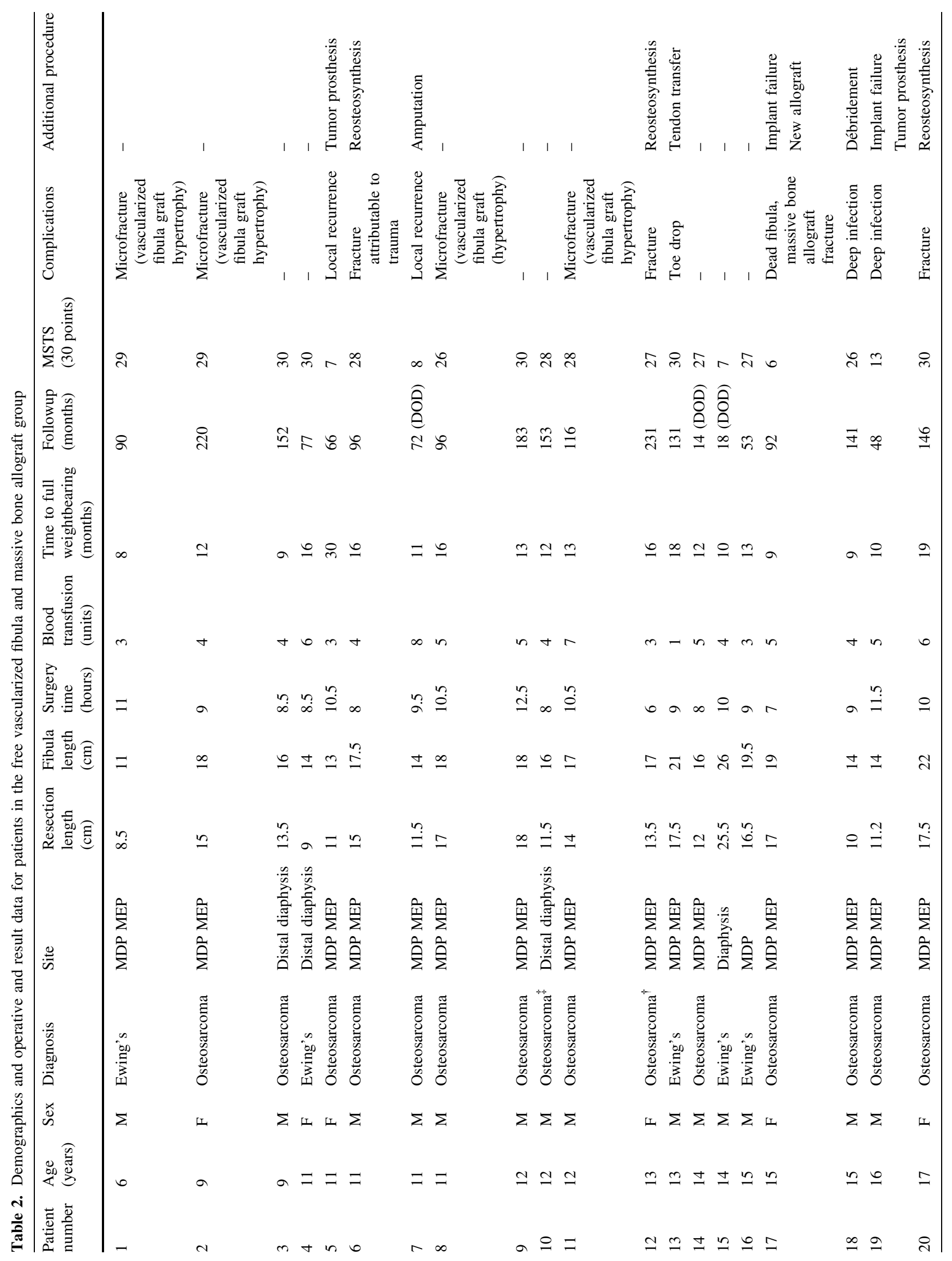


transfer for toe drop (1). With the numbers available, we observed no difference in the proportion of patients experiencing surgical complications between those treated with free vascularized fibula grafts and those treated with pedicled grafts (eight of 22 [36\%] versus nine of 25 [36\%] respectively; $p=0.605$ ). Microfracture of the massive bone allograft was observed on CT scans of eight patients (four in each group), with subsequent hypertrophy of the inlaid vascularized fibula graft leading to endosteal fusion, and not requiring any further intervention. Delayed union was observed in one patient in the pedicled vascularized fibula group, and iliac crest bone grafting was performed. Four patients in each group sustained a fracture (one in each group, attributable to significant trauma) requiring reosteosynthesis in all but one patient who was treated conservatively with an above knee cast. One patient with a fracture had signs of fibula hypertrophy, but underwent reosteosynthesis owing to implant instability. A dual-onlay allograft was used for reosteosynthesis in one patient with fracture whose inlaid pedicled fibula graft had not shown signs of hypertrophy or fusion with the allograft. Toe drop in the donor limb requiring tendon transfer was required in one patient in the free vascularized fibula group. Deep infection was treated with débridement alone in one patient, and a two-stage procedure with a modular proximal tibia tumor endoprosthesis was performed in one patient in the free fibula group. One patient in the pedicled fibula group underwent removal of the biologic construct followed by insertion of a cement spacer and distraction osteogenesis with an Ilizarov frame.

The overall MSTS functional score was $24 \pm 8$ in 45 patients who were available for evaluation. Two patients died before 1 year and did not have documented MSTS scores. With the numbers available, we observed no difference in mean MSTS scores between those treated with free vascularized fibula grafts and those treated with pedicled grafts ( $24 \pm 9$ versus $25 \pm 8$; mean difference, 0.48 ; 95\% CI, 0.54-4.6; $\mathrm{p}=0.858$ ). With the numbers available, we observed no difference in mean time to full weightbearing ambulation between those treated with free fibula grafts and those treated with pedicled fibula grafts (13 \pm 5 months versus $11 \pm 5$ months; mean difference, 1.5 months; 95\% CI, $1.5-4.5$ months; $\mathrm{p}=0.323$ ).

The mean surgical time was longer in the free vascularized fibula and massive bone allograft group at $9.4 \pm 1.7$ hours compared with that of the pedicled vascularized fibula and massive bone allograft group at $5.7 \pm 1.3$ hours (mean difference, 3.73 hours; 95\% CI, 2.8-4.6 hours; $\mathrm{p} \leq$ 0.001 ). With the numbers available, we could not detect a difference in mean intra- and postoperative blood transfusion between the two groups at $4.6 \pm 1.6$ and $3.8 \pm 1.6$ units respectively (mean difference, 0.73 units; 95\% CI, $0.30-1.76$ units; $\mathrm{p}=0.16$ ) (Table 4). 


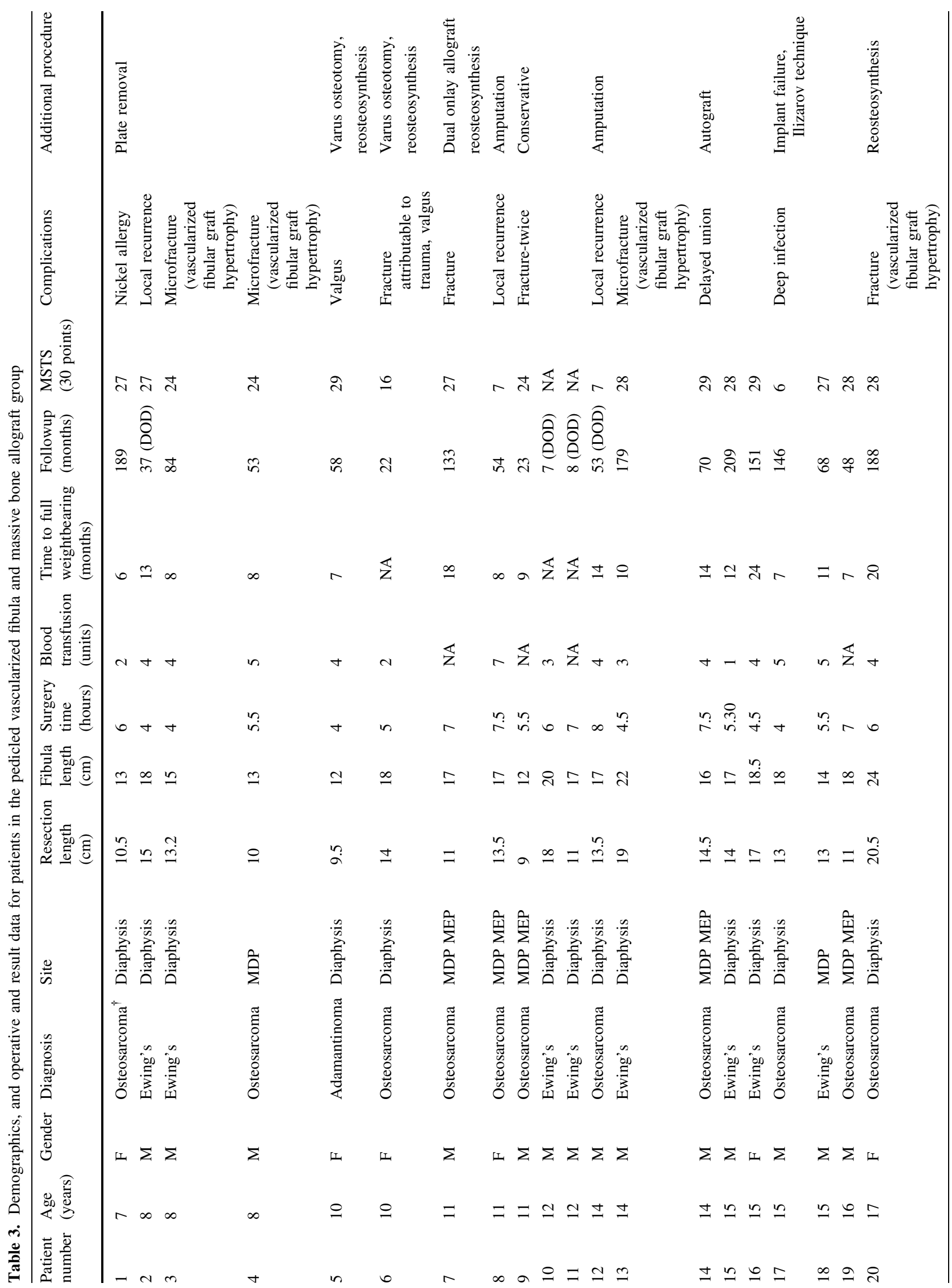




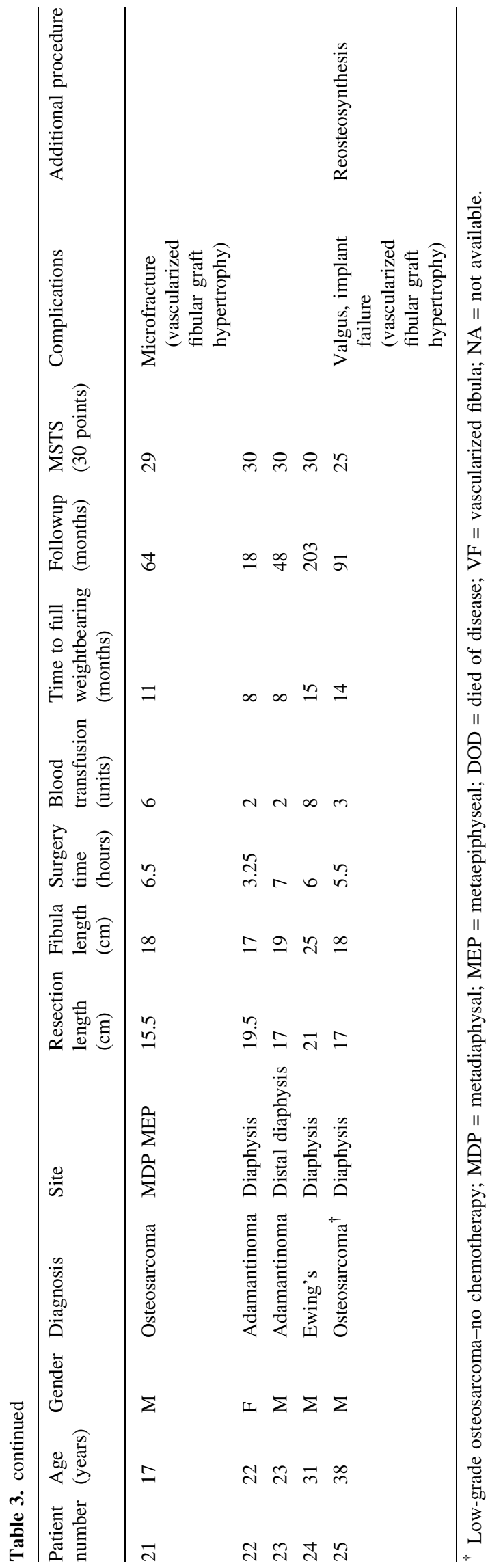

\section{Discussion}

Intercalary resection of the tibia with adequate margins and reconstruction can be performed owing to advanced imaging techniques and constantly improving surgical expertise. Biologic intercalary reconstruction for primary and salvage surgeries in sarcomas involving massive bone allograft and supplemental vascularized fibula graft can attempt to mitigate the complications associated with use of either an allograft alone (delayed union, nonunion, fracture, and failure of fixation, especially in defects larger than $18 \mathrm{~cm}$ requiring further revision surgery) or vascularized fibula alone (prone to loss of viability secondary to microvascular reconstruction, fatigue fracture, and morbidity owing to the longer duration of surgery) [17]. The combination of a massive bone allograft and vascularized fibula construct provides immediate structural support, gradual revascularization, and incorporation $[7,18,24]$. Owing to morbidity associated with contralateral free vascularized fibula harvesting, a pedicled vascularized fibula graft from the ipsilateral side can be harvested with little added morbidity [11, 29]. Studies on massive bone allograft supplemented with vascularized fibula graft for tibia intercalary reconstructions in primary or salvage procedures are well documented $[2,4,6,7]$. Several studies involving middle and distal tibia reconstructions with pedicled fibula grafts alone have shown good results with the advantage of being less expensive, a technically easier procedure, associated with minimal complications, and with a predictable long-term clinical outcome [2, 11-13, 19, 30]. Other studies involving vascularized fibula and massive bone allograft constructs have shown similar advantages, focusing either on only free vascularized fibula $[4,7,10,21-23,31]$ or pedicled vascularized fibula with massive bone allografts [9, 29]. However, to our knowledge, there are no published studies on indications or comparisons for free versus pedicled vascularized fibula grafts supplementing massive bone allografts in tibia diaphyseal sarcomas. We reasoned that if the results of both techniques were equivalent, that the pedicled grafts had advantages such as avoiding harvesting a fibula from the contralateral leg, avoidance of an anastomosis, and shorter operative time.

This retrospective study has several limitations. The two groups of patients with tibia diaphyseal sarcoma who received vascularized fibula grafts (free or pedicled) were not randomized with respect to osteotomy sites; therefore, the two groups may not be directly comparable. The study size for comparison is limited owing to the combined relative rarity of tibia diaphyseal malignancies treated with specific biologic reconstruction, making statistical comparisons difficult. In addition, the diagnoses were heterogeneous (osteosarcoma, Ewing's sarcoma, and adamantinoma), and not all patients received chemotherapy which may affect bone healing. However, the strengths of this 
Fig. 3A-H (A) This AP radiograph shows the proximal tibia in an 11-year-old girl with osteosarcoma. (B) An immediate postoperative AP radiograph shows the free vascularized fibula and massive bone allograft construct after intraepiphyseal osteotomy. (C) Fracture of the allograft construct with varus deformity is seen on this AP view radiograph obtained at 8 years followup. (D) An axial CT section shows the fracture of the construct at 8 years followup. The allograft has sustained multiple fractures and the fibula is radiodense without change in density or size indicating a nonviable fibula graft. Postoperative (E) $\mathrm{AP}$ and (F) lateral view radiographs obtained after revision surgery show this patient's construct with fresh allograft and dual plate reosteosynthesis after removal of the original construct consisting of the nonviable free vascularized fibula graft with fractured allograft. (G) AP and (H) lateral view radiographs obtained at the 13-year followup show that the graft has healed after the revision at 8 years with a new freshfrozen allograft and dual-plate osteosynthesis. The patient has visible arthritic changes of the knee.
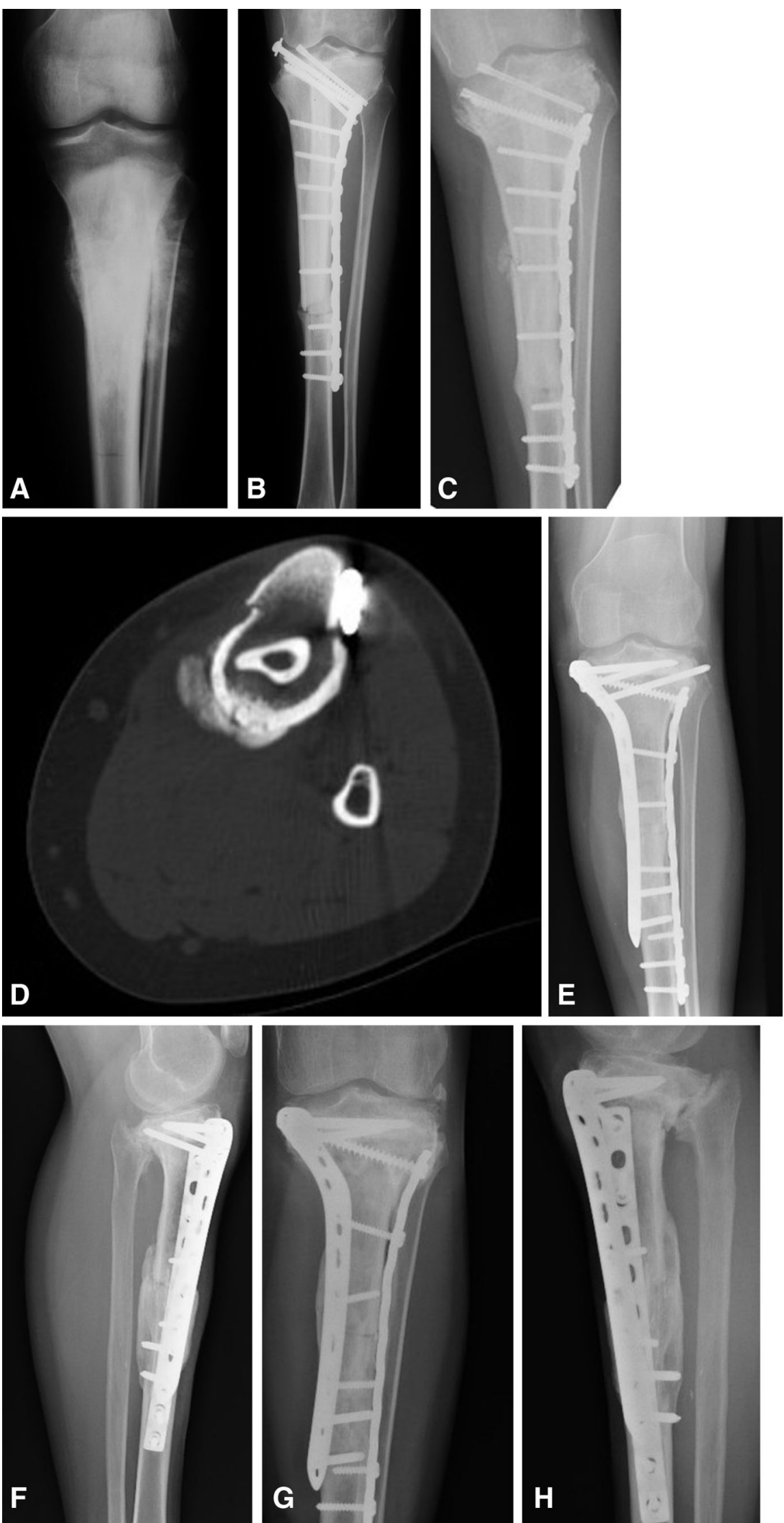
Table 4. Comparison of results between groups

\begin{tabular}{|c|c|c|c|}
\hline Variable & $\begin{array}{l}\text { Free vascularized fibula-massive } \\
\text { bone allograft group } \\
(\mathrm{n}=22)\end{array}$ & $\begin{array}{l}\text { Pedicled vascularized } \\
\text { fibular-massive bone } \\
\text { allograft group } \\
(\mathrm{n}=25)\end{array}$ & $\begin{array}{l}\mathrm{p} \text { Value } \\
0.05\end{array}$ \\
\hline Age (years) & $13.27 \pm 4.2$ & $14.96 \pm 7.2$ & 0.338 \\
\hline Resection (cm) & $14.65 \pm 4.0$ & $14.41 \pm 3.5$ & 0.830 \\
\hline Harvested fibula $(\mathrm{cm})$ & $17.68 \pm 3.9$ & $17.34 \pm 3.2$ & 0.747 \\
\hline Surgical time (hours) & $9.41 \pm 1.7$ & $5.68 \pm 1.3$ & $<0.001$ \\
\hline Blood transfusion (units) & $4.64 \pm 1.6$ & $3.90 \pm 1.7$ & 0.16 \\
\hline Time to partial weightbearing (months) & $2.45 \pm 0.6$ & $2.80 \pm 1.3$ & 0.266 \\
\hline Time to full weightbearing (months) & $12.95 \pm 5.2$ & $11.45 \pm 4.7$ & 0.323 \\
\hline Complications & 8 & 9 & 0.605 \\
\hline Infection & 2 & 1 & \\
\hline Delayed union & 0 & 1 & \\
\hline Fracture & 4 & $4 *$ & \\
\hline Mechanical complications/deformity & 1 & 2 & \\
\hline Neurovascular & 1 & 0 & \\
\hline Implant related/allergy & 0 & 1 & \\
\hline Additional procedures & 8 & 8 & 0.543 \\
\hline Autograft & 0 & 1 & \\
\hline Reosteosynthesis/removal & 5 & 6 & \\
\hline Change of construct & 1 & 1 & \\
\hline Débridement & 1 & 0 & \\
\hline Tendon surgery & 1 & 0 & \\
\hline Local recurrence & 2 & 3 & 0.747 \\
\hline Tumor prosthesis & 1 & 0 & \\
\hline Amputation & 1 & 2 & \\
\hline Metastases & 4 & 2 & 0.398 \\
\hline Died of disease & 3 & 4 & 1.00 \\
\hline Followup (months) & $110.66 \pm 58.2$ & $88.10 \pm 65.1$ & 0.223 \\
\hline \multirow[t]{2}{*}{ MSTS functional score (30 points) } & $(\mathrm{n}=22)$ & $(\mathrm{n}=23)^{\dagger}$ & 0.858 \\
\hline & $23.81 \pm 8.8$ & $24.26 \pm 7.6$ & \\
\hline
\end{tabular}

* One fracture was managed conservatively; MSTS = Musculoskeletal Tumor Society score; ${ }^{\dagger}$ two patients died of disease before the MSTS assessment.

study were that we did not detect any major differences in the characteristics (age, tumor location, tumor resection size, size of harvested vascularized fibula graft, and osteosynthesis) of the two groups. Free vascularized fibula and massive bone allograft reconstructions were performed after intraepiphyseal resections (18 performed during the early part of the study period), whereas pedicled vascularized fibula and massive bone allograft reconstructions were performed for diaphyseal resections. This difference in harvesting free vascularized fibula grafts for intraepiphyseal resections was attributed to the length and mobilization of the pedicle being perceived as difficult in pedicled vascularized fibula grafts and the subsequent risk of vascular compromise. However, with time, we learned this was not a true limitation. Seven pedicled vascularized fibula grafts were harvested and mobilized for intraepiphyseal resections during the second decade of the study based on our early unpublished results with experience of pedicled vascularized fibula grafts and with results from other studies [11-13, 23, 28-30]. This observation and technique were encouraging considering there was no difference in either length of resection or harvested vascularized fibula graft in both groups, including excess vascularized fibula length. We believe that an intraepiphyseal resection in the proximal or distal tibia is not a contraindication to harvest pedicled and graft pedicled vascularized fibula with massive bone allograft, but it does require considerable experience in mobilizing the vascular pedicle to limit complications. 
The $8.5 \%$ incidence of local recurrence is comparable to the recurrence rate reported by Abed et al. [1]. We acknowledge that because our patients had a mixture of high- and low-grade osteosarcoma, Ewing's sarcoma, and adamantinoma, the overall survival figures are difficult to compare with those of published studies, and should be interpreted with caution. We think it is important to note however, that neither local relapse nor survival seemed to be impaired by using either of these techniques of reconstruction.

Ninety-one percent of allografts supplemented with vascular fibula with allograft construct in our study patients survived at 10 years compared with rates ranging from $79 \%$ to $93.5 \%$ with up to 9 years of followup in other studies [7, 8, 20-23, 26, 29]. The overall complication rate of $34 \%$ was comparable to rates reported for vascularized fibula and massive bone allograft reconstruction [7, 10, 20]. We found no published studies with which to compare our results of supplemental free versus pedicled fibula grafts for massive bone allografts. The overall fracture rate was $17 \%$ for the incidence of fracture of the vascularized fibula and massive bone allograft (excluding microfractures), with reported results ranging between $0 \%$ and $33 \%$ [31]. There were no differences in the rate of allograft fracture (macrofracture requiring surgery or microfracture not requiring surgery) between the two groups of patients and this did not seem to be related to the type of slot made in the allograft to seat the fibular graft. Lateral plating was avoided in free fibula grafts owing to the risk of failure of anastomosis with the anterior tibialis bundle, which was not the case in pedicled fibula grafts. In both groups the grafted vascularized fibula showed evidence of change in density and resorption (viability) on regular followup CT. Scintigraphy was used to assess graft construct viability in several studies $[1,23,26]$, however we preferred to use radiographs and $\mathrm{CT}$ scans of the surgically treated limb to assess the progress of the vascularized fibula bone allograft construct. Three observations on the healing pattern of vascularized fibula graft seen on serial CT scans have been reported [9, 25]. The first is that progressive thinning and incorporation of the vascularized fibula graft with massive bone allograft (endosteal bony bridge) was observed in 17 patients who had free vascularized fibula grafts and 21 who had pedicled vascularized fibula grafts. A second pattern of microfractures of massive bone allograft and subsequent fibula hypertrophy and endosteal graft incorporation with massive bone allograft was observed in four patients in each group. None of these eight patients needed additional procedures. The third pattern is where the vascularized fibula graft does not change in density or size and shows fractures without healing, termed graft necrosis presumably owing to failed blood supply to the fibular graft. This was observed in one free vascularized fibula graft that was revised with graft removal, fresh massive bone allograft, and osteosynthesis. An ipsilateral pedicled fibula graft was not used for revision in this situation owing to the short stature of the patient with a short residual harvestable fibula that would have increased morbidity (tumor resection length being $17 \mathrm{~cm}$ ), and thereby was preserved to retain mechanical support provided by the ipsilateral fibula. Based on the above behavior patterns, we could assess the viability of the fibula and plan a revision of the construct.

An interesting finding was the occurrence of one varus deformity in a patient with a free vascularized fibula and massive bone allograft and two valgus deformities in patients in the pedicled vascularized fibula and massive bone allograft group. All of these occurred in patients with diaphyseal tibia reconstructions and were treated by corrective osteotomy and osteosynthesis. The incidence of delayed union was less than $1 \%$, comparatively lower than reported incidences ranging from $4 \%$ to $33.3 \%$ [7, 8, 20-23, 26, 29]. Partial weightbearing was allowed by $2.6 \pm 1$ months and full weightbearing ambulation was permitted by $12 \pm 5$ months, similar to those reported for 2 months [31] and 13 months [20-23, 26] on average, respectively. The low incidence of delayed union and subsequent deformities between our two groups we believe is attributable to central placement of the vascularized fibula graft in the massive bone allograft shell followed by adequate and stable osteosynthesis [7, 9, 34]. Revision of a vascularized fibula and massive bone allograft construct was performed in one patient in each group owing to one dead fibula in the free vascularized fibula group and an uncontrolled infection in the pedicled vascularized fibula group. Thus, even though complications resulted in an average of one additional procedure per three patients, the final salvage of the vascularized fibula and massive bone allograft construct was acceptable and seldom results in ultimate failure, change of the construct, or amputation. The infection rate was comparable to the $7.5 \%$ rate reported by Capanna et al. [7] in their series of massive bone allograft supplemented with free vascularized fibula grafts and was comparatively lower than rates in series of allograft or vascularized fibula alone [4, 6, 12, 13], and this led to a change of the construct in two of our patients (one in each group). The frequency of complications in this study overall, and between the two fibula graft groups with additional procedures used to manage these complications did not differ, but a larger study might reveal some differences.

The overall MSTS scores for our patients were comparable to those of massive bone allograft with vascularized fibula ranging between 23 and 27 [4, 7, 8, 20, 22, 23], whereas one study, in which only pedicled versus free fibula grafts alone were compared, showed poor MSTS scores and overall outcomes for the pedicled graft group 
[6]. With the numbers we had, we could not show a difference in MSTS scores between the free fibula and the pedicled fibula groups.

We observed that harvesting a free vascularized fibula graft took longer and that the patients had an operative time more than 3 hours longer than that of patients treated with pedicled vascularized fibula grafts. We did not find a difference in the need for blood transfusion, with the number of patients we had in our study. Theoretically, longer operative time increases the risk of infection, need for blood transfusion, and transfusion-related reactions [31]. However, there was no significant difference in the rate of infection between the two groups. The decreased time with use of pedicled vascularized fibula grafts is likely because it does not require additional time for a microvascular anastomosis, thereby effectively reducing overall operating time [11, 23, 29].

Intercalary reconstruction of tibia sarcomas with massive bone allograft supplemented with vascularized fibula graft provides predictable results with complications in up to onethird of cases that are salvageable, preserving the original construct. We believe a pedicled vascularized fibula supplementing an allograft can perform well for diaphyseal resections and selected intraepiphyseal resections, and has the apparent advantages, compared with free fibula transfers, of being a simpler technique and having a shorter operative time while achieving comparable outcomes. Comparative technical ease, shorter surgical time, avoidance of additional microvascular anastomosis, and avoidance of surgery on the contralateral leg are notable advantages of pedicled vascularized fibula over free fibula to supplement allografts when used in intercalary tibia resections.

\section{References}

1. Abed YY, Beltrami G, Campanacci DA, Innocenti M, Scoccianti G, Capanna R. Biological reconstruction after resection of bone tumours around the knee: long-term follow-up. J Bone Joint Surg Br. 2009;91:1366-1372.

2. Atkins RM, Madhavan P, Sudhakar J, Whitwell D. Ipsilateral vascularised fibular transport for massive defects of the tibia. $J$ Bone Joint Surg Br. 1999;81:1035-1040.

3. Bullens PH, Minderhoud NM, de Waal Malefijt MC, Veth RP, Buma P, Schreuder HW. Survival of massive allografts in segmental oncological bone defect reconstructions. Int Orthop. 2009;33:757-760.

4. Campanacci DA, Puccini S, Caff G, Beltrami G, Piccioli A, Innocenti M, Capanna R. Vascularised fibular grafts as a salvage procedure in failed intercalary reconstructions after bone tumour resection of the femur. Injury. 2014;45:399-404.

5. Campanacci L, Manfrini M, Colangeli M, Alí N, Mercuri M. Long-term results in children with massive bone osteoarticular allografts of the knee for high-grade osteosarcoma. J Pediatr Orthop. 2010;30:919-927.

6. Cao QL, Wang Z, Yu XW, Teng Y, Zhang QJ, Cai JY. [Comparison of long bone repair in tibia by vascularized fibular grafting of different sides][in Chinese]. Zhongguo Xiu Fu Chong Jian Wai Ke Za Zhi. 2002;16:109-111.

7. Capanna R, Campanacci DA, Belot N, Beltrami G, Manfrini M, Innocenti M, Ceruso M. A new reconstructive technique for intercalary defects of long bones: the association of massive allograft with vascularized fibular autograft. Long-term results and comparison with alternative techniques. Orthop Clin North Am. 2007;38:51-60, vi.

8. Ceruso M, Falcone C, Innocenti M, Delcroix L, Capanna R, Manfrini M. Skeletal reconstruction with a free vascularized fibula graft associated to bone allograft after resection of malignant bone tumor of limbs. Handchir Mikrochir Plast Chir. 2001;33:277-282.

9. Ceruso M, Taddei F, Bigazzi P, Manfrini M. Vascularised fibula graft inlaid in a massive bone allograft: considerations on the biomechanical behaviour of the combined graft in segmental bone reconstructions after sarcoma resection. Injury. 2008;39(suppl 3):S68-S74.

10. Chang DW, Weber KL. Use of a vascularized fibula bone flap and intercalary allograft for diaphyseal reconstruction after resection of primary extremity bone sarcomas. Plast Reconstr Surg. 2005;116:1918-1925.

11. Chung YK, Chung S. Ipsilateral island fibula transfer for segmental tibial defects: antegrade and retrograde fashion. Plast Reconstr Surg. 1998;101:375-382; discussion 383-384.

12. Ebeid W, Amin S, Abdelmegid A, Refaat Y, Ghoneimy A. Reconstruction of distal tibial defects following resection of malignant tumours by pedicled vascularised fibular grafts. Acta Orthop Belg. 2007;73:354-359.

13. El-Sherbiny M. Long term behavior of pedicled vascularized fibular grafts in reconstruction of middle and distal tibia after resection of malignant bone tumors. J Egypt Natl Cancer Inst. 2008;20:187-195.

14. Enneking WF, Dunham W, Gebhardt MC, Malawar M, Pritchard DJ. A system for the functional evaluation of reconstructive procedures after surgical treatment of tumors of the musculoskeletal system. Clin Orthop Relat Res. 1993;286:241-246.

15. Ferrari S, Ruggieri P, Cefalo G, Tamburini A, Capanna R, Fagioli F, Comandone A, Bertulli R, Bisogno G, Palmerini E, Alberghini M, Parafioriti A, Picci, P, Bacci G. Neoadjuvant chemotherapy with methotrexate, cisplatin, and doxorubicin with or without ifosfamide in nonmetastatic osteosarcoma of the extremity: an Italian sarcoma group trial ISG/OS-1. J Clin Oncol. 2012;30: 2112-2118.

16. Ferrari S, Sundby Hall K, Luksch R, Tienghi A, Wiebe T, Fagioli F, Alvegard TA, Brach Del Prever A, Tamburini A, Alberghini M, Gandola L, Mercuri M, Capanna R, Mapelli S, Prete A, Carli M, Picci P, Barbieri E, Bacci G, Smeland S. Nonmetastatic Ewing family tumors: high-dose chemotherapy with stem cell rescue in poor responder patients: results of the Italian Sarcoma Group/Scandinavian Sarcoma Group III protocol. Ann Oncol. 2011;22:1221-1227.

17. Frisoni T, Cevolani L, Giorgini A, Dozza B, Donati DM. Factors affecting outcome of massive intercalary bone allografts in the treatment of tumours of the femur. J Bone Joint Surg Br. 2012;94:836-841.

18. Fuchs B, Ossendorf C, Leerapun T, Sim FH. Intercalary segmental reconstruction after bone tumor resection. Eur J Surg Oncol. 2008;34:1271-1276.

19. Hatori M, Ayoub KS, Grimer RJ, Carter SR, Tillman RM. The two-stage ipsilateral fibular transfer for tibial defect following tumour excision. Sarcoma. 2000;4:27-30.

20. Innocenti M, Abed YY, Beltrami G, Delcroix L, Manfrini M, Capanna R. Biological reconstruction after resection of bone tumors of the proximal tibia using allograft shell and 
intramedullary free vascularized fibular graft: long-term results. Microsurgery 2009;29:361-372.

21. Jager T, Journeau P, Dautel G, Barbary S, Haumont T, Lascombes $\mathrm{P}$. Is combining massive bone allograft with free vascularized fibular flap the children's reconstruction answer to lower limb defects following bone tumour resection? Orthop Traumatol Surg Res. 2010;96:340-347.

22. Li J, Wang Z, Guo Z, Chen GJ, Fu J, Pei GX. The use of allograft shell with intramedullary vascularized fibula graft for intercalary reconstruction after diaphyseal resection for lower extremity bony malignancy. J Surg Oncol. 2010;102:368-374.

23. Li J, Wang Z, Guo Z, Chen GJ, Li SW, Pei GX. The use of massive allograft with intramedullary fibular graft for intercalary reconstruction after resection of tibial malignancy. $J$ Reconstr Microsurg. 2011;27:37-46.

24. Manfrini M, Gasbarrini A, Malaguti C, Ceruso M, Innocenti M, Bini S, Capanna R, Campanacci M. Intraepiphyseal resection of the proximal tibia and its impact on lower limb growth. Clin OrthopRelat Res. 1999;358:111-119.

25. Manfrini M, Vanel D, De Paolis M, Malaguti C, Innocenti M, Ceruso M, Capanna R, Mercuri M. Imaging of vascularized fibula autograft placed inside a massive allograft in reconstruction of lower limb bone tumors. AJR Am J Roentgenol. 2004;182:963-970.

26. Moran SL, Shin AY, Bishop AT. The use of massive bone allograft with intramedullary free fibular flap for limb salvage in a pediatric and adolescent population. Plast Reconstr Surg. 2006;118:413-419.
27. O’Flanagan SJ, Stack JP, McGee HM, Dervan P, Hurson B. Imaging of intramedullary tumour spread in osteosarcoma: a comparison of techniques. J Bone Joint Surg Br. 1991;73:9981001.

28. Ozaki T, Fujiwara K, Kunisada T, Ito T, Kawai A, Inoue H. Reconstruction with ipsilateral fibula transfer with pasteurized bone after excision of bone sarcoma of the tibia. Sarcoma. 2004;8:97-102.

29. Ozaki T, Hillmann A, Wuisman P, Winkelmann W. Reconstruction of tibia by ipsilateral vascularized fibula and allograft: 12 cases with malignant bone tumors. Acta Orthop Scand. 1997;68:298-301.

30. Puri A, Subin BS, Agarwal MG. Fibular centralisation for the reconstruction of defects of the tibial diaphysis and distal metaphysis after excision of bone tumours. J Bone Joint Surg Br. 2009;91:234-239.

31. Rabitsch K, Maurer-Ertl W, Pirker-Frühauf U, Wibmer C, Leithner A. Intercalary reconstructions with vascularised fibula and allograft after tumour resection in the lower limb. Sarcoma. 2013;2013:160295. doi: 10.1155/2013/160295.

32. Thompson RC Jr, Pickvance EA, Garry D. Fractures in largesegment allografts. J Bone Joint Surg Am. 1993;75:1663-1673.

33. Weber KL. What's new in musculoskeletal oncology. $J$ Bone Joint Surg Am. 2005;87:1400-1410.

34. Wood MB. Free vascularized fibular grafting-25 years' experience: tips, techniques, and pearls. Orthop Clin North Am. 2007;38:1-12, v. 